\title{
Efficient Design and Implementation of LTE Downlink Control Information Decoder
}

\author{
Mohamed S. Abo Zeid, Khaled M. Elsayed, Mohamed E. Nasr, and Salah El Deen A. Khamis
}

\begin{abstract}
The decoding of Downlink Control Information (DCI) in LTE is based upon a process that is defined as a blind decoding which depends on a number of decoding attempts on a number of Physical Downlink Control Channel (PDCCH) candidate locations for a number of defined DCI formats. In this paper, two proposed designs for DCI decoder are presented. The first decodes based upon monitoring PDCCH candidate locations serially. This design can perform the whole 44 decoding attempts within $87.3 \%$ of the whole OFDM symbol time with extended cyclic prefix. So, it's well suited for Multicast/Broadcast over Single Frequency Network (MBSFN) subframes. However, this design doesn't meet the worst-case time of an LTE OFDM symbol with normal cyclic prefix. Therefore, a second design is proposed that exploits parallelism to enable fast blind decoding process. The proposed parallel design proves efficiency in meeting decoding time constraints besides, consuming a little power with a proven efficiency in utilization area perspective. The proposed parallel design performs all of 44 decoding attempts in a time of $7.8 \mu$ s which is only $11.7 \%$ of an LTE useful OFDM symbol time with a logic power consumption of only $17000 \mu \mathrm{W}$. The proposed designs are simulated using Modelsim 6.4a and implemented in Plan Ahead 14.4 in $28 \mathrm{~nm}$ technology, Virtex7 FPGA kit of part number XC7V2000T, which is characterized by high performance and large capacity.
\end{abstract}

Index Terms-LTE, UESSS, CSS, DCI, PDCCH, UE.

\section{INTRODUCTION}

Long Term Evolution (LTE) is a fourth generation wireless high speed broadband technology developed by the third generation partnership project (3GPP) to provide high data rates, low latency, high mobility, reduced costs and compatibility with existing systems besides packetoptimized radio access technology supporting flexible bandwidth deployments [1].

The increasing demands of today's user poses a challenge in the design of recent communication systems like 3GPP LTE to perform fast processing of user data. Fast processing of user data requires reliable delivery of the data and the associated control signaling that specifies the format of the data and the location and timing of the radio resources allocated to the user. To accomplish high data rates, the LTE standard requires the usage of instantaneous adaptive

Manuscript received July 10, 2013; revised September 3, 2013

Mohamed S. Abo Zeid, Mohamed E. Nasr, and Salah El Deen A. Khamis are with Electronics and Electrical Communications Dept., Faculty of Engineering, Tanta University, Tanta and 31527, Egypt (e-mail: m1_saeed@yahoo.com, salah.khamis@f-eng.tanta.edu.eg, mnasr@feng.tanta.edu.eg).

Khaled M. Elsayed is with Electronics and Electrical Communications Dept., Faculty of Engineering, Cairo University, Giza and 12613, Egypt (email: author@lamar.colostate.edu). modulation and coding techniques to accommodate the channel quality conditions [2]. However, this poses a challenge of proper signaling of these adaptive parameters to the UE on a separate control channel which results in increasing the channel bandwidth required for signaling and hence reducing the bandwidth available for the user's traffic [3]. An alternative approach is that the UE is neither informed about its intended DCI format nor its location within the control region of subframe. Therefore, The UE performs a number of decoding attempts to obtain the intended control format. This process is the blind decoding process [4]. Such process has the advantage of reducing the amount of resources needed for control information transmission while simultaneously increasing the reliable delivery of data and control information [5].

The 3GPP LTE specifications define the candidate locations that the UE shall monitor, instead of monitoring all locations within the control region, to reduce the UE power consumption and the timing needed to accomplish that monitoring [6]. These defined set of resources in which each UE shall monitor its control information are called search spaces.

The standard of LTE, also, defines two search spaces. UE-Specific Search Space (UESSS) where its candidate locations are monitored by specific UE and it is used for transmission of control information that configures specific UE. On the other hand, Common Search Space (CSS) where its candidate locations are monitored by all UEs in the serving cell and it is used for transmission of common control information for all eNodeB's UEs [7].

In this paper two proposed designs for DCI decoder are presented. The first monitors the PDCCH candidate locations in a serial manner. Although, it is proved efficiency from decoding time perspective with subframes comprising of OFDM symbols with extended cyclic prefixes, it is being verified that it does not meet the worstcase time of an LTE OFDM symbol with normal cyclic prefix. Therefore, a second design is proposed that exploits parallelism to enable fast blind decoding process. The design also has proven efficiency in meeting time requirements of the decoding process and also good utilization area and power efficiency.

The rest of the paper is organized as follows. Section II gives information about LTE frame structure. Section III describes the basics of LTE downlink control channels. Section IV deals with blind decoding process. Section V provides the proposed system design model. The serial decoder is presented in section VI while the parallel design is presented in Section VII. In Section VIII results and discussion are provided followed by conclusions in Section IX. 


\section{LTE FRAME STRUCTURE}

LTE supports two frame types FDD and TDD, in this paper FDD is the adopted one where one LTE radio frame duration is $10 \mathrm{~ms}$ consisting of $10 \mathrm{sub}$ frames, each of $1 \mathrm{~ms}$ duration. Each subframe is divided into two slots, each of $0.5 \mathrm{~ms}$ duration which includes seven OFDM symbols for normal cyclic prefix and six OFDM symbols for extended cyclic prefix, the FDD frame structure is shown in Fig. 1 [8].

According to LTE specifications, a subcarrier spacing $=15 \mathrm{KHz}$ corresponds to a useful OFDM symbol time $=$ $(1 /$ subcarrier spacing $) \approx 66.7 \mu \mathrm{s}$ is defined [8]. The overall OFDM symbol time consists of useful OFDM symbol time plus cyclic prefix time. With normal cyclic prefix the whole OFDM symbol time is $71.4 \mu$ s compared to $83.4 \mu$ s with extended cyclic prefix.

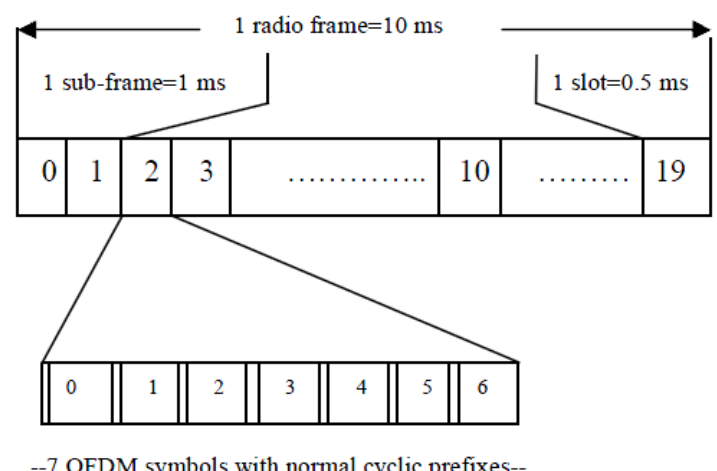

Fig. 1. FDD Frame Structure.

\section{LTE CONTROL CHANNEL PRELIMINARIES}

The LTE downlink includes 3 physical control channels. The Physical Control Format Indicator Channel (PCFICH) carries the Control Format Indicator (CFI) which determines the number of OFDM symbols (1, 2 or 3 OFDM symbols) in $1 \mathrm{~ms}$ sub-frame used for transmission of downlink control information. Physical Downlink Control Channel (PDCCH) is the heart of downlink control channels as it carries the Downlink Control Information (DCI). DCI includes the uplink scheduling grants and downlink scheduling assignments [1]. It contains, also, the type of modulation being used for Physical Downlink Shared Channel (PDSCH), which carries UE's downlink data, besides other control information. Physical H-ARQ Indicator Channel (PHICH) carries the downlink ACK/NACK information associated with uplink data transmission [9].

The basic building unit of $\mathrm{PDCCH}$ is the Control Channel Element (CCE), where each CCE consists of nine Resource Element Groups (REGs). Each REG consists of four physical Resource Elements (REs) with each RE carrying Quadrature Phase Shift Keying (QPSK) symbol corresponding to one OFDM symbol and one subcarrier. Hence, one CCE includes 36 QPSK symbols equivalent to 72 bits [10].

Each PDCCH consists of one CCE or an aggregation of CCEs. CCEs aggregation is a form of repetition coding. In accordance to LTE specifications, there are only four aggregation levels supported. So, four PDCCH formats one for each CCE aggregation level as shown in Table I are defined [11]. CCEs are consecutively used and numbered in sequence. The total number of CCEs in the control region of sub-frame depends on the bandwidth of the system and the aggregation level being used for transmission of control information which depends exactly on the channel condition experienced by the UE [12].

\begin{tabular}{|c|c|c|c|}
\multicolumn{4}{|c|}{ TABLE I: LTE PDCCH FORMATS } \\
\hline \multirow{2}{*}{$\begin{array}{c}\text { PDCCH } \\
\text { FORMATs }\end{array}$} & \multicolumn{3}{|c|}{ LTE PDCCH FORMATS } \\
\cline { 2 - 4 } & No. of CCEs & $\begin{array}{c}\text { No. } \\
\text { of REGs }\end{array}$ & No. of PDCCH bits \\
\hline 0 & 1 & 9 & 72 \\
1 & 2 & 18 & 144 \\
2 & 4 & 36 & 288 \\
3 & 8 & 72 & 576 \\
\hline
\end{tabular}

Each PDCCH carries DCI. Different DCI formats are supported in LTE. Each of these DCI formats contains the minimum payload required for a particular scenario which reduces the signaling overhead. The supported DCI formats are shown in Table II [11].

\begin{tabular}{|c|c|}
\hline $\begin{array}{c}\text { DCI } \\
\text { Format }\end{array}$ & DCI Format Purpose \\
\hline 0 & Used for scheduling of PUSCH \\
\hline 1 & Transmission of assignments of PDSCH \\
\hline $1 \mathrm{~A}$ & Compact scheduling of PDSCH \\
\hline $1 \mathrm{~B}$ & PDSCH assignments for rank 1 transmission \\
\hline $1 \mathrm{C}$ & Used for very compact transmission of PDSCH \\
\hline $1 \mathrm{D}$ & $\begin{array}{l}\text { Used for compact signaling of PDSCH assignments } \\
\text { for multi-user MIMO. }\end{array}$ \\
\hline 2 & $\begin{array}{l}\text { PDSCH assignments for closed loop MIMO } \\
\text { operation. }\end{array}$ \\
\hline $2 \mathrm{~A}$ & $\begin{array}{l}\text { PDSCH assignments for open loop MIMO } \\
\text { operation. }\end{array}$ \\
\hline $3 \& 3 \mathrm{~A}$ & $\begin{array}{l}\text { Transmit power control commands for multiple } \\
\text { users for PUCCH and PUSCH with 2-bits and 1-bit } \\
\text { power adjustments, respectively. }\end{array}$ \\
\hline
\end{tabular}

The LTE standard defines that the number of control information bits of each DCI format depends mainly on the assigned downlink resource blocks (DL Bandwidth) and uplink resource blocks (UL Bandwidth). They typically obtained from transmission of basic system information on broadcast channel $(\mathrm{BCH})$. The standard also, defines that DCI formats $0,1 \mathrm{~A}, 3$ and $3 \mathrm{~A}$ have the same payload size of control information bits [5].

\section{The LTE PDCCH Blind DeCoding PROCESS}

In PDCCH blind decoding process, the UE using UESpecific Search Space (UESSS), from Table III, shall monitor $6 \mathrm{PDCCH}$ candidate locations in aggregation level $1(L=1)$, and $6 \mathrm{PDCCH}$ candidate locations in aggregation level 2, in addition to, $2 \mathrm{PDCCH}$ candidate locations in $L=4$ and $L=8$. So, a total of $16 \mathrm{PDCCH}$ candidate locations shall be monitored in case of UESSS. The UE shall attempt to decode all DCI formats in these candidate locations which would pose strict challenges on timing constraints and power usage. However, 3GPP LTE specifications define that each UE may receive only 2 DCI formats per Transmission Time Interval (TTI) of $1 \mathrm{~ms}$. One reference DCI format is always to be received which is DCI format $0 / 1 \mathrm{~A}$. As $0 / 1 \mathrm{~A}$ DCI formats have the same payload size of control information bits with one flag bit for differentiation between 
them so, only one blind decoding attempt is required for decoding them per PDCCH candidate location [10]. In addition to that attempt, one more decoding attempt per PDCCH candidate location for one of other DCI formats 1 , $1 \mathrm{~B}, 1 \mathrm{D}, 2,2 \mathrm{~A}$ is needed where only one of them every TTI is assigned to the UE. This DCI format is corresponding to the configured transmission mode that is signaled from higher layer (RRC signaling) to the UE. Hence, In the UESSS, a total of $16 \times 2=32$ blind decoding attempts are required to monitor all $\mathrm{PDCCH}$ candidate locations for the assigned two DCI format types per TTI.

\begin{tabular}{|c|c|c|c|}
\multicolumn{4}{c|}{ TABLE III: LTE SEARCH SPACES [7] } \\
\hline \multirow{3}{*}{$\begin{array}{c}\text { Search } \\
\text { Space } \\
\text { Type }\end{array}$} & \multicolumn{3}{|c|}{ LTE Search Spaces } \\
\cline { 2 - 4 } & $\begin{array}{c}\text { Aggregation } \\
\text { level } \\
\text { (L) }\end{array}$ & $\begin{array}{c}\text { Size [in } \\
\text { CCEs] }\end{array}$ & $\begin{array}{c}\text { Number of } \\
\text { PDCCH } \\
\text { Candidates }\end{array}$ \\
\hline \multirow{2}{*}{ UESSS } & 1 & 6 & 6 \\
& 2 & 12 & 6 \\
& 4 & 8 & 2 \\
& 8 & 16 & 2 \\
\hline \multirow{2}{*}{ CSS } & 4 & 16 & 4 \\
& 8 & 16 & 2 \\
\hline
\end{tabular}

As indicated in Table III, for Common Search Space (CSS), PDCCH candidate locations are defined in aggregation levels 4 and 8 only. For $L=4$, there are 4 $\mathrm{PDCCH}$ candidate locations, and for $L=8$ only $2 \mathrm{PDCCH}$ candidate locations are defined. So, in total, 6 blind decoding attempts to monitor all candidate locations for Common Search Space are needed. The UE monitors in Common Search Spaces DCI formats 0, 1A, 3, 3A and 1C. Since DCI formats $0 / 1 \mathrm{~A}$ and $3 / 3 \mathrm{~A}$ have the same payload size, one blind decoding attempt per PDCCH candidate location is needed to decode them. Additionally, one more decoding attempt is, also, needed per PDCCH candidate location for DCI format $1 \mathrm{C}$ which is used for scheduling of broadcast control information which includes paging, random access response and system information block transmissions [5]. As a result, total of $6 \times 2=12$ blind decoding attempts are used to check all PDCCH candidate locations for the 2 DCI formats assigned every TTI for the Common Search Space. As a consequence of that a total of $12+32=44$ blind decoding attempts are required to monitor all PDCCH candidate locations in both the CSS and UESSS for the assigned DCI formats every TTI.

According to LTE specifications, The UE monitors only one CSS at each of the aggregation levels 4 and 8 and one UESSS at each of the aggregation levels 1, 2, 4, 8. The UESSS and CSS may overlap [7].

\section{SYSTEM DESIGN MODEL}

Fig. 2 shows the block diagram of LTE DCI encoder at eNodeB. The DCI control information bits are firstly, appended with 16 cyclic redundancy check (CRC) parity bits for error detection purposes. Also, to save additional payload, the resultant $16 \mathrm{CRC}$ parity bits are masked the Radio Network Temporary Identifier (RNTI) of the particular intended UE . This will also help to check if this DCI is intended to that UE or not. After that, the resultant
DCI bits with the 16 appended RNTI masked CRC bits undergo tail-biting convolutional coder (TBCC) with rate $1 / 3$ and constraint length of 7 [13]. A rate matching block is then used to obtain the desired code rate. Finally, PDCCH candidate locations defined from UESSS for particular UE and CSS are determined to place these DCI formats in appropriate candidate locations with the desired code rate. Fig. 3 shows the reverse process for these operations at the UE.

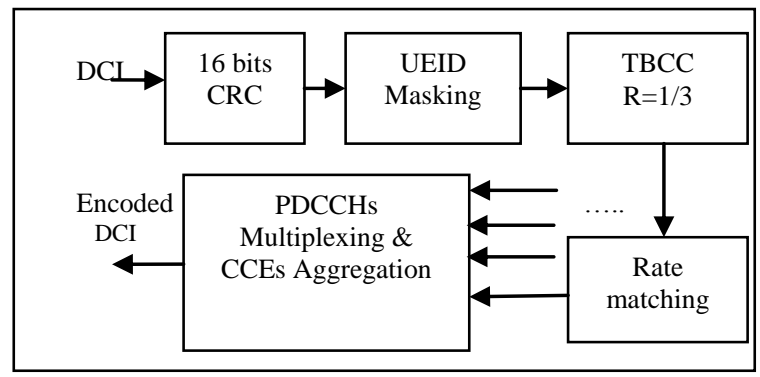

Fig. 2. Block Diagram of LTE DCI Encoder.

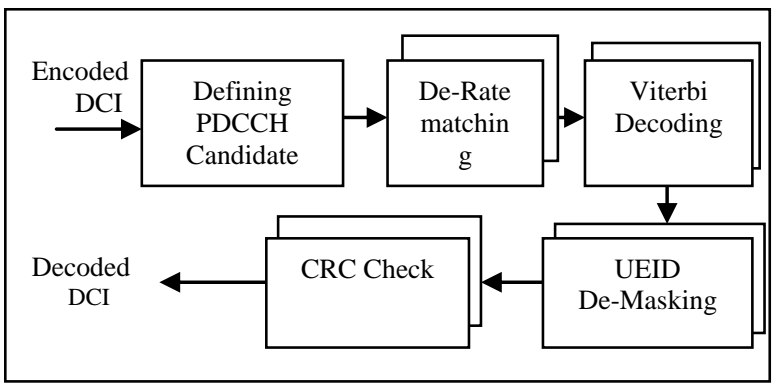

Fig. 3. Block Diagram of LTE DCI Decoder.

The procedure for blind decoding process performed at the UE can be summarized as shown in Fig. 4.

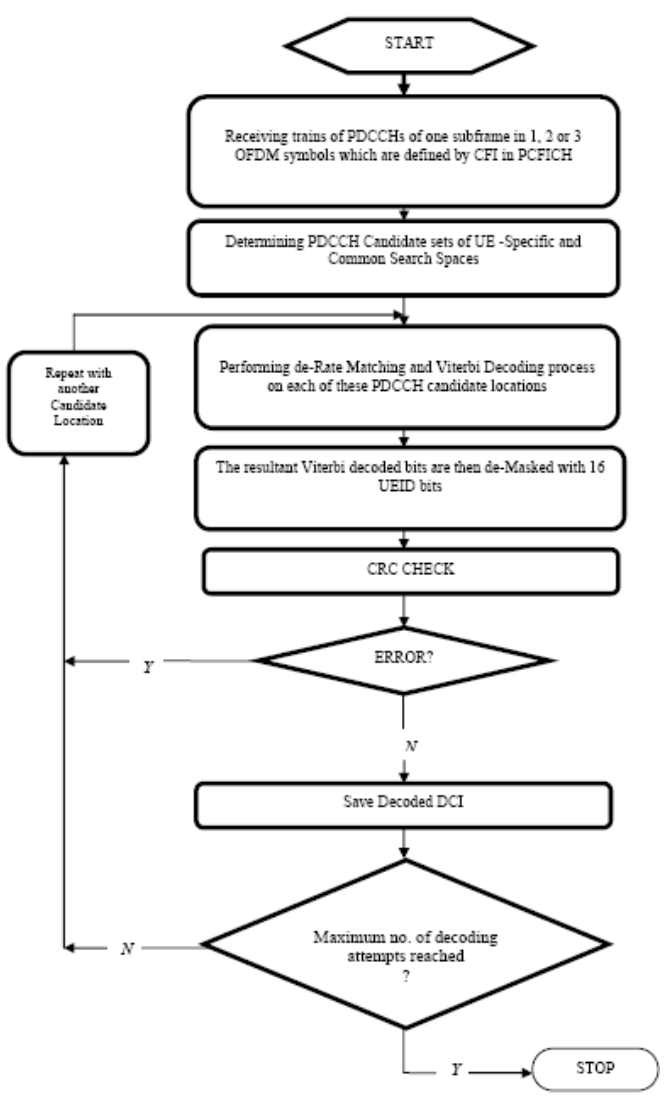

Fig. 4. Design model flow chart. 


\section{The Proposed SERIAL DESIGN}

The Proposed serial design consists of a chain of functional blocks each of it is performing a specific function in the blind decoding process as shown in the following sections.

\section{A. Defining PDCCH Candidate Locations}

The PDCCH candidate locations are defined in terms of CCEs using UE-Specific Search Space and Common Search Space hashing functions as shown in (1) [12]:

$$
\begin{gathered}
S_{k}(L)=L \times\left\{\left(Y_{k}+m\right) \bmod \left[N_{(c c e, k)} / L\right]\right\}+i \\
Y_{k}=\left(A \times Y_{k-1} \bmod D\right)
\end{gathered}
$$

$S_{k}(L)$ is UESSS of sub-frame $k$ at aggregation level $L$ where $L \in\{1,2,4,8\}$ and $i=0,1,2, \ldots, L-1$.

$N_{(c c e, k)}$ is the total number of CCEs in the control region of sub-frame $k$ and $m=0,1,2, \ldots, M(L)$, where $M(L)$ is the number of monitored PDCCH candidates in the given search space corresponding to aggregation level $L$ [12], as shown in Table III.

$Y_{k}$ is given from (2) where $A=39827, D=65537$ and $k=$ $\left(n_{s} / 2\right)$ where $n_{s}$ is the slot number within a radio frame. $Y_{-1}$ is initially assigned with the Radio Network Temporary Identifier (RNTI) of the intended UE.

$S_{k}(L)$ is also, the hashing function for CSS of sub-frame k at aggregation level $L$ where $L \in\{4,8\}$ and $i=0,1, \ldots, L$ - 1, provided that $Y_{k}$ is set to 0 for only these two aggregation levels $L=4$ and $L=8$ [7].

\section{B. Clocked RAM}

The objective of this RTL block is to select the PDCCH bits from each of the defined PDCCH candidate locations at each of the aggregation levels for both UESSS and CSS. It also, provides a memory to store the selected bits from the multiplexed PDCCHs bits train and after finishing blind decoding process of each of PDCCH candidate locations, the selected bits of the second PDCCH candidate location are provided for the decoding process. This will be performed at every positive edge of the assigned clock frequency. Hence, its name is clocked RAM.

\begin{tabular}{|c|c|}
\hline \multirow{2}{*}{$\begin{array}{l}\text { Number of } \\
\text { columns } \\
\text { C }\end{array}$} & Inter-column permutation pattern \\
\hline & $\langle P(0), P(1), \ldots \ldots \ldots \ldots, P(C-1)\rangle$ \\
\hline 32 & $\begin{array}{l}<1,17,9,25,5,21,13,29,3,19,11,27,7,23,15, \\
31,0,16,8,24,4,20,12,28,2,18,10,26,6,22, \\
14,30>\end{array}$ \\
\hline
\end{tabular}

\section{De-Rate Matcher}

In this block De-Rate matching process is performed assuming a rate matching of $1 / 3$ is performed at the transmitter. So, the received PDCCH bits consist of first parity bits then second one followed by the third parity one. Each of these parity bits at eNodeB are separately interleaved using sub-block interleaver, that is performing 32 columns permutation operation, as shown in Table IV. Therefore, a reverse process at the UE is required. Each parity is firstly de-interleaved and then the three parity bits are concatenated to construct the PDCCH bits which are ready for Viterbi decoding process.

where $C$ is the number of columns of permutation matrix and it is fixed while the number of rows is variable such that the minimum integer satisfy

$$
N \leq(R \times C)
$$

where $N$ is the number of bits in each of the first, second and third convolutionaly coded parity streams. $R$ and $C$ are the number of rows and columns of permutation matrix, respectively.

\section{Viterbi Decoding}

In the proposed serial design, four Viterbi blocks are used to decode the codewords received corresponding to four PDCCH formats $0,1,2$, and 3. The Viterbi blocks decode the bits encoded with TBCC with rate $1 / 3$, constraint length $=7$ and generator polynomials represented in octal form by [13]

$$
G 0=(133)_{8} \quad G 1=(171)_{8} \quad G 2=(165)_{8}
$$

One Viterbi block is responsible for decoding of the payload size of PDCCH format 0 of an aggregation of one CCE which contains 72 bits. With assumption of rate matching of $1 / 3$ and after elimination of dummy bits with processing of three bits at a time, so, 24 decoding stages are required. Hence; the resultant number of decoded bits is 24 bits. According to the proposed designed Viterbi, 24 counts a counter with an array of $24 \times 64$ bit registers are required for proper storage of survivor path decisions for each of 64 states at each of 24 decoding stages. Therefore, 24 clock cycles to feed forward 3 bits every clock cycle to construct trellis are needed. In addition, two more clock cycles are needed, one for defining state with minimum path metric and the other for tracing back to obtain the decoded output sequence. So, a total of 26 clock cycles to accomplish of Viterbi decoding process for payload of 72 bits are needed.

Another viterbi decoder is responsible for decoding of payload size of PDCCH format 1 which of aggregation level of 2 of Control Channel Elements (CCEs) which is equal to 144 bits. So, with processing of codeword of 3 bits at a time 48 decoding stages are needed for accurate decoding. As a result 48 clock cycles are required to build viterbi trellis with three bits at a time and one more clock cycle for selecting best state with minimum path metric besides, one additional clock cycle for tracing back. So, in total, a result of $48+2=50$ clock cycles to accomplish the decoding process of payload size of PDCCH format1.

The third Viterbi decoder is used for PDCCH format 2 which consists of an aggregation of 4 CCEs with a payload size of 288 bits. The Viterbi decoding process uses 96 counts a counter with an array of $96 \times 64$ bit registers. Therefore, 96 clock cycles are needed to build the decoding stages of Viterbi. Besides, two clock cycles are needed for defining state with minimum path metric and tracing back. So, in total, 98 clock cycles are required to perform such decoding operation.

The last Viterbi block is used for decoding the payload of $\mathrm{PDCCH}$ format 3 which is of an aggregation of $8 \mathrm{CCEs}$ results in payload size of 576 bits. So, decoding of such 
code words require a total of 192 decoding stages. So, the number of clock cycles required up to obtaining the decoded output sequence is 194 clock cycles. This format is the worst case to decode with this large amount of time required for just Viterbi decoding process. This time is repeated serially 4 times where $4 \mathrm{PDCCH}$ candidate locations are defined in PDCCH format 3. Two locations for each of UESSS and CSS are defined.

Also, in the proposed Viterbi design, a clock gating technique which allows only the trace-back block to be switched on after finishing of the processing of the entire received codewords. Besides, the use of counter with clock gating technique allows only one 64 bits register of the memory array for storing survivor path decision for each state to be on at a time. These methods in designing will result in reducing the dynamic power dissipation which is an important concern for saving the limited UE battery power.

\section{E. UEID De-Masking}

After performing the Viterbi decoding of received $\mathrm{PDCCH}$ format corresponds to PDCCH candidate location defined from hashing function, UEID de-masking is performed according to (4) [12]:

$$
q(i)=(b(i)+c(i)) \bmod 2
$$

where $b$ is the 16 bits CRC parity sequence and $c$ is 16 bits RNTI of the intended UE. As the LTE standard defines that, 3/3A DCI formats are masked with different RNTIs other than C-RNTI which is used to mask 0/1A DCI formats, therefore, the UEID is variable in the RTL according to the required RNTI for De-Masking function.

\section{F. CRC Check}

CRC check block is a combinational logic that performs 16 bits CRC check in only one clock cycle to reduce the total amount of delayed time required for blind decoding process. Using 16-bits CRC generator polynomial in (5) that is defined from 3GPP LTE specifications [13]

$$
g_{\text {crc16 }}(Z)=\left[Z^{16}+Z^{12}+Z^{5}+1\right]
$$

If the generated CRC parity bits similar to the received CRC parity bits, So this DCI format assigned to that UE and saved in memory for performing the required operations on PDSCH based on its' control field information. Otherwise, so this DCI format is not intended to that UE or it may be corruptly received with too many errors. In both cases, this payload is neglected and not being considered.

\section{The Proposed PARALlEl Design}

In the proposed parallel design, the basic blocks of the proposed serial design are used with some modifications in designing of these blocks, especially, Viterbi blocks which are the heart of blind decoding process that causes the large amount of decoding delay in the blind decoding process.

Instead of using a single decoding window which results in increasing the decoding delay, a high speed Viterbi processing of the received code words is proposed. It is based on the technique of sliding window method where the decoding process considers independent blocks that can be processed simultaneously in forward and backward directions [14]. With this method, blocks of data can be processed independently which causes unlimited concurrency that results in speeding the decoding process of long stream of bits within a small amount of time. With the Viterbi decoding condition of that the decoding window size must be greater than or equal to five times of constraint length [15], also, as 3GPP LTE specifications define the constraint length of TBCC to be 7. So, a decoding window of 35 stages or greater can be used effectively for decoding process with negligible decoding performance degradation. Hence, the Viterbi decoder for PDCCH format 1 can be used as the basic decoding window for both $\mathrm{PDCCH}$ format 2 and $\mathrm{PDCCH}$ format 3. For PDCCH format 2 the number of concurrent block is limited to two, while for PDCCH format 3 , a number of 4 concurrent blocks provide an efficient decoding performance.

Also, the clocked RAM used in parallel design is different from that of the one used in the proposed serial design. Parallelism is utilized to speed up the blind decoding process. The clocked RAM stores only the selected bits for the first $3 \mathrm{PDCCH}$ candidate locations while other one is being used to store the selected bits for the remaining 3 PDCCH candidate locations simultaneously. This is the case for $6 \mathrm{PDCCH}$ candidate locations. While for only $4 \mathrm{PDCCH}$ candidate locations for PDCCH format 3 which is the basic part of large decoding delay. One clocked RAM is used to store the selected bits for only 2 first candidate locations. At the same time, another one is used for storing the selected bits for the other 2 PDCCH candidate locations. So, instead of performing one decoding attempt at a time, with this method two decoding attempts are performed simultaneously at a time.

Also, the PDCCH candidate locations for UESSS are treated independently of the CSS candidate locations thus achieving parallelism in their decoding.

\section{RESULTS AND DISCUSSION}

\section{A. Proposed Serial Design Simulation Results}

The resultant simulation of the proposed serial search design is shown in Fig. 5, while Fig. 6 shows its Register Transfer Logic (RTL) diagram. The variable 'CLK' is the input port for the assigned clock frequency; 'UEID' is the CRNTI for the UE which performs blind decoding to search for its assigned DCI formats. In the simulation, it is assigned to 14. Variable 'ncce' is input indicating the number of CCEs in the control region used for estimating clocked RAM which stores PDCCH bits of PDCCH format 1. CLK4 is assigned for clocked RAM storing of bits of PDCCH format 2. Finally, CLK8 for RAM that PDCCH candidate locations (UESSS - CSS hashing functions). In the simulation, it is assigned to 20. 'TTI' is the input port of sub-frame number. 'PDCCHs' is also an input port for the received train of PDCCHs bits. ' res_UESS_com' is the input port for resetting to the initial state after every a complete blind decoding process. As shown from Fig. 5, CLK11, CLK2, CLK4, CLK8 are obtained from dividing the clock frequency assigned to the system for providing serially, the bits of every PDCCH candidate location, after completing 
the previously decoded candidate one. CLK11 is the assigned clock for clocked RAM storing PDCCH bits of $\mathrm{PDCCH}$ format 0 . While CLK2 is assigned for stores $\mathrm{PDCCH}$ bits of PDCCH format 3. As being stated, it's the slowest one since the large decoding delay of single decoding window. The first two check variables are 'Check1dci1', 'Check1dci2' which are output ports for indicating whether there are DCI formats (DCI1, DCI2), for each PDCCH decoded candidate of PDCCH format 0 , intended for the UE or not. If there are, so check with logic 0 is resulted and the correctly decoded DCI bits are stored in a memory which its size depends on the length of the decoded DCI bits. These two check variables in the simulation waveform followed by 'Check2dci1', 'Check2dci2', 'Check4dci1', 'Check4dci2', 'Check8dci1' and
'Check8dci2', respectively. These check variables indicate whether there are DCI formats (DCI1, DCI2) for each PDCCH decoded candidate of PDCCH format 1 , format 2 and format 3, respectively, intended for the UE or not. It's cleared from the simulation waveform, when 'check1dcil' indicates zero logic so, a successful decoding attempt is resulted and the monitored $\mathrm{PDCCH}$ payload is intended to the UE. The variable 'originalctrl_bits1DCI1' stores these DCI bits of PDCCH of format 0 , and it can be clearly noticed that this action is synchronized with 'CLK11' cycle. Also, the stored DCI bits are changed every clock cycle of CLK11 based on the blindly decoding of the monitored PDCCH candidate. The same operation occurs with PDCCHs of formats 1,2 and 3.

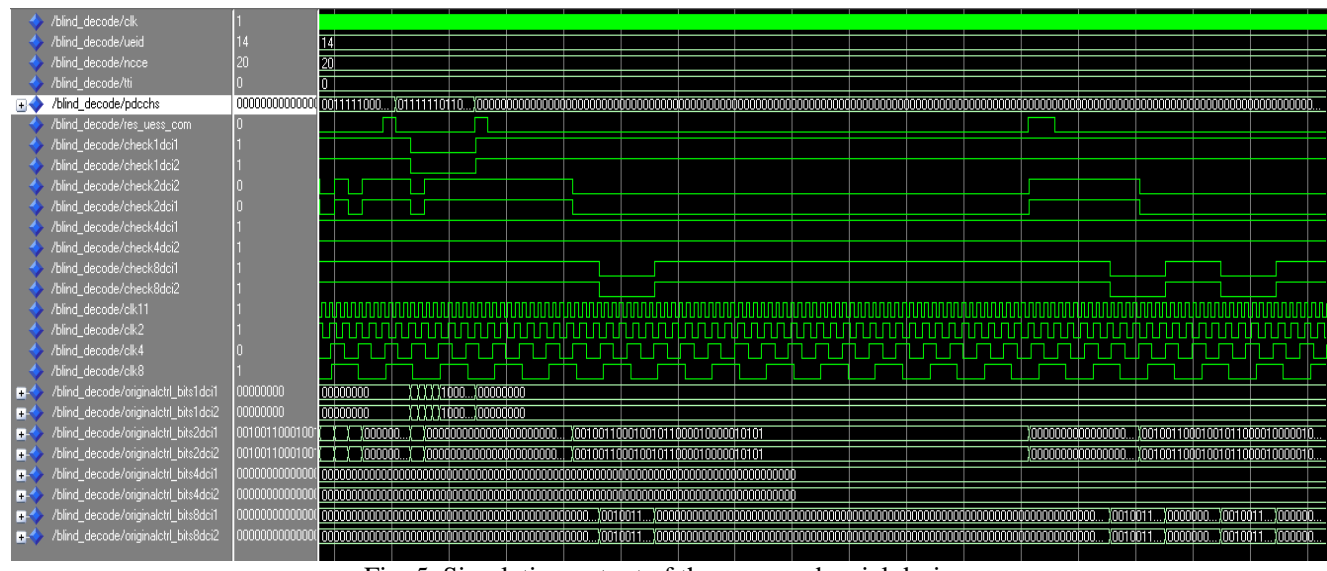

Fig. 5. Simulation output of the proposed serial design.

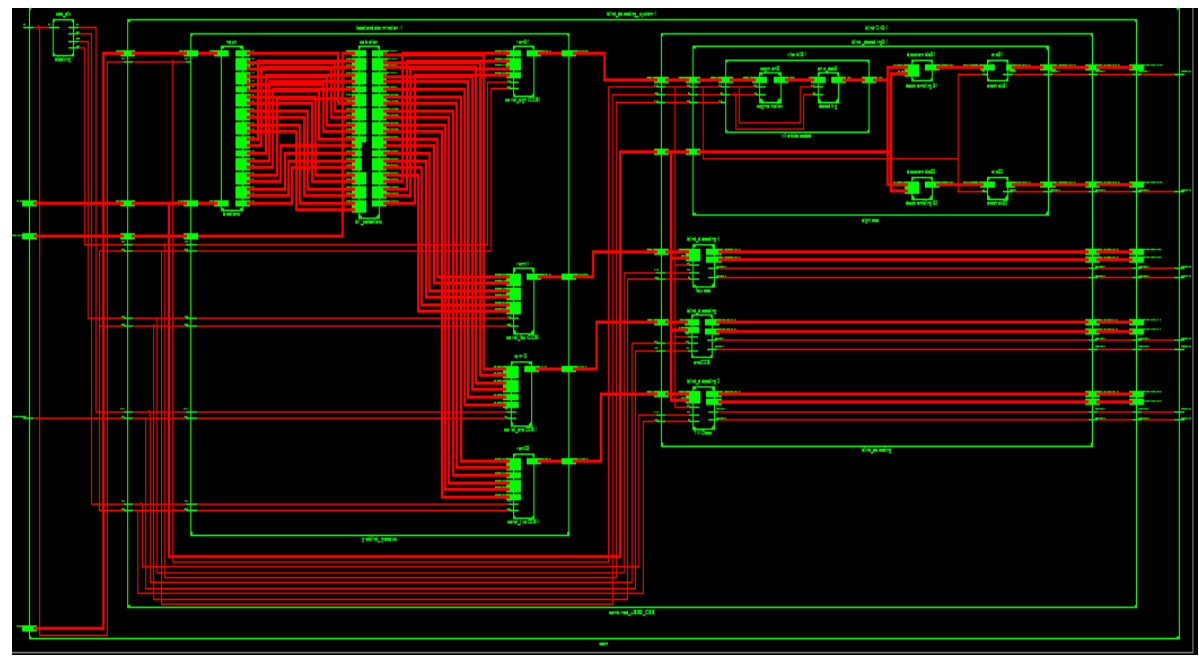

Fig. 6. RTL Diagram of the proposed serial design 。

\section{B. Timing Calculation of the Proposed Serial Design}

The maximum operating frequency resulted from the synthesis report for the proposed serial search design is 10.565 MHz which is equivalent to a minimum clock period of $94.649 \mathrm{~ns}$. As previously mentioned, for the system to perform 44 blind decoding attempts, it must search for the four candidate locations of PDCCH format 3 which consists of an aggregation level of $8 \mathrm{CCEs}$ with a total of PDCCH received bits of 576 bits. So, the total number of clock cycles required to complete one blind decoding of one candidate location of PDCCH format 3 is 194 clock cycles for Viterbi decoding plus one clock cycle for CRC checking.
With minimum clock period of 94.649 ns. So the total time required for one blind decoding attempt of PDCCH format 3 is $94.649 n s \times 195=18456.555 n s=18.4 \mu \mathrm{sec}$. From UESSS and CSS, the total number of PDCCH candidate locations for PDCCH format 3 is 4 (2 candidate locations for UESSS and 2 for CSS). So a total of 4 decoding attempts will be performed for PDCCH format 3. So, 4 times of the computed period for serially processing each of $\mathrm{PDCCH}$ candidates are needed.

As a result, the total period of time required to complete all of blind decoding attempts for PDCCH format 3 is 18.4 $\mu s \times 4=73.6 \mu \mathrm{sec}$. With this computed time to accomplish all of 44 blind decoding attempts and with the LTE defined 
useful OFDM useful symbol time of $66.7 \mu \mathrm{sec}$, the proposed serial search design is not time efficient at all. As the time required to perform all of 44 blind decoding attempts is $110.3 \%$ of useful OFDM symbol time which is more than one OFDM symbol time. However, it can perform the whole 44 decoding attempts within $87.3 \%$ of the whole OFDM symbol time (useful OFDM symbol time + extended cyclic prefix time) which makes it an efficient design for decoding of only MBSFN sub-frames with extended cyclic prefixes.

\section{Utilization Area and Power Consumption of the Proposed Serial Design}

The resultant proposed serial design is synthesized and implemented on virtex7 FPGA kit of part number XC7V2000T. The utilization area report is shown in Fig. 7.

The power consumption of the Register Transfer Logic (RTL) is estimated using Xilinx Power Estimator (XPE). The basic part of power consumption is the dynamic power which depends heavily on the switching activity. The consumed dynamic power is $30000 \mu \mathrm{W}$ with only $2 \%$ of them for logic power while the remaining part is consumed from clocking activity, as shown in Fig. 8.

\begin{tabular}{|l|r|r|r|r|}
\hline \multicolumn{5}{|l|}{ Device Utilization Summary (estimated values) } \\
\hline Logic Utilization & Used & Avallable & Utilization & -1 \\
\hline Number of Slice Registers & 30349 & 2433200 & $1 \%$ \\
\hline Number of Slice LUTs & 205928 & 1221600 & $3 \%$ \\
\hline Number of fully used LUTFF pairs & 7287 & 228990 & $73 \%$ \\
\hline Number of bonded IOBS & 626 & 850 & $4 \%$ \\
\hline Number of BUFG/BUFGCTRLs & 6 & 128 & $0 \%$ \\
\hline Number of DSP48E1s & 20 & 2160 & \\
\hline
\end{tabular}

Fig. 7. FPGA virtex7 utilization area report.

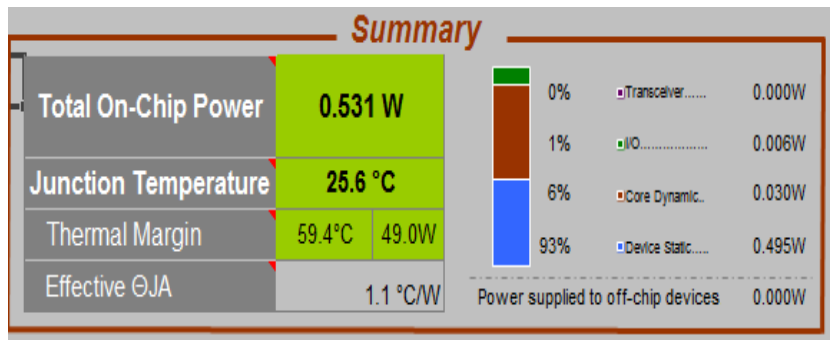

Fig. 8. Total on-chip power.

As shown from Fig. 8, The leakage power is $495000 \mu \mathrm{W}$, while the core dynamic power is only $6 \%$ of total on chip power of $531000 \mu \mathrm{W}$ under the temperature constraints that. The power consumed from IO ports is $6000 \mu \mathrm{w}$. The logic power is only $12000 \mu \mathrm{W}$ while clock power is $18000 \mu \mathrm{W}$, as shown from Fig. 9.

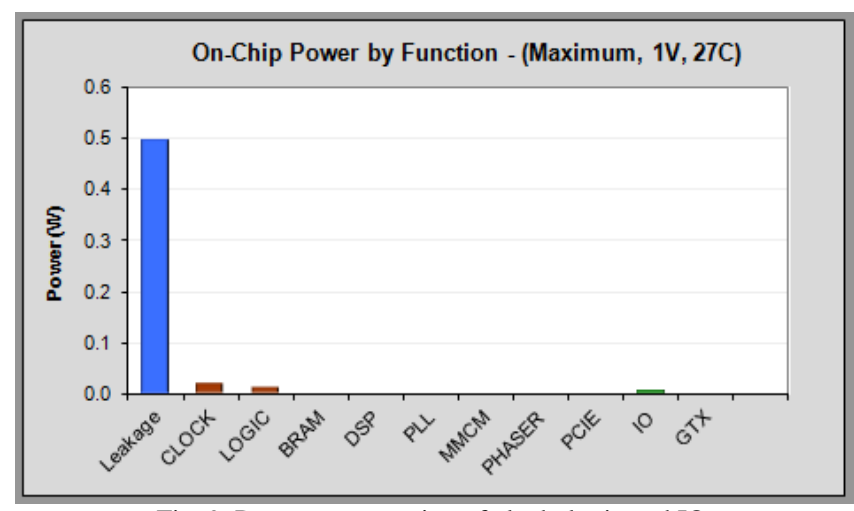

Fig. 9. Power consumption of clock, logic and IO.

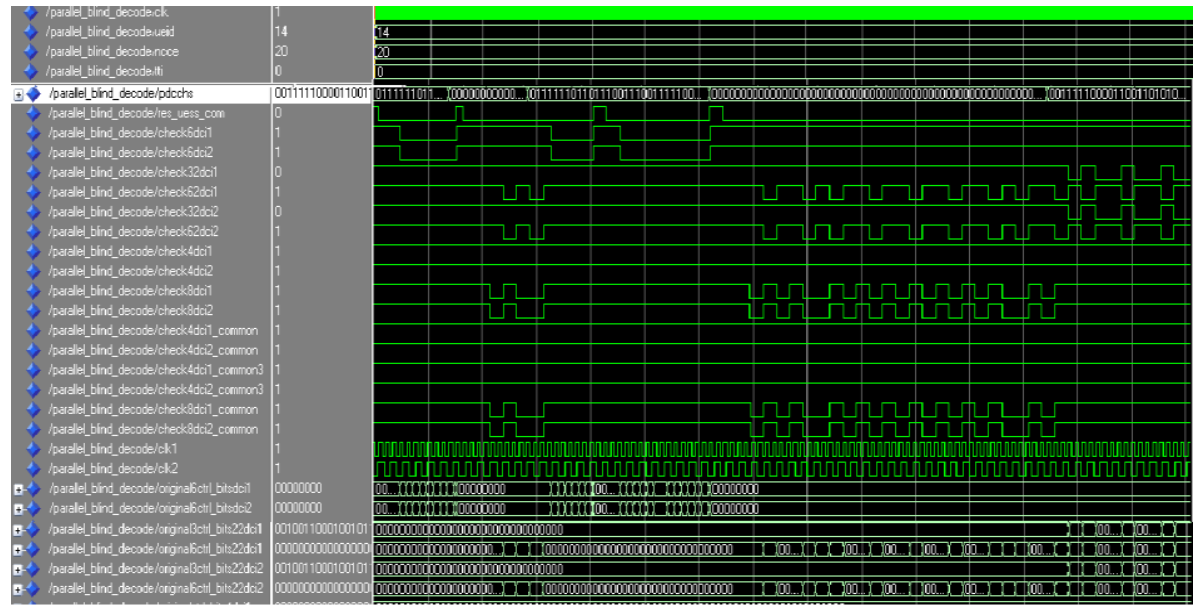

Fig. 10. Simulation output of the proposed parallel design.

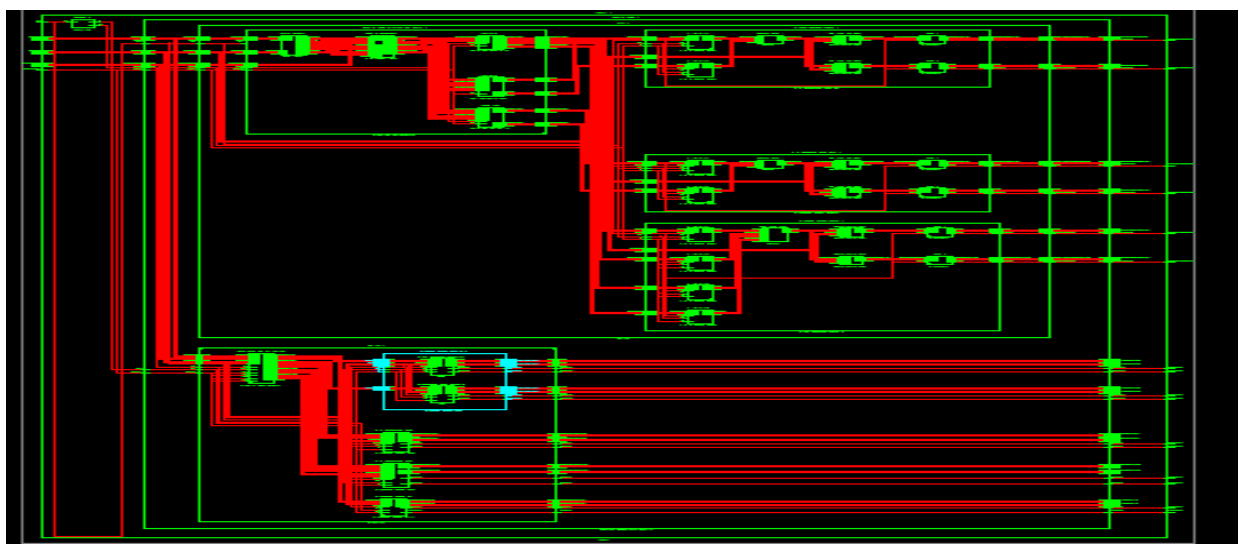

Fig. 11. RTL Diagram of the proposed parallel design. 


\section{Proposed Parallel Design Simulation Results}

The resultant simulation waveform is shown in Fig.10. In the simulation, different PDCCH train bits are assumed with each of it 2 different DCI formats are assumed. Also, Different PDCCH formats are assumed for testing, as seen some times DCI bits of PDCCH format 0 are obtained. In other times, DCI bits of PDCCH format 1 are resulted.

The variable 'CLK' is the input port for the assigned clock frequency. 'Ueid' is the input port for assigning C-RNTI of the intended UE. It can be any RNTI assigned to UE. 'Ncce' is also, an input port for assigning the number of Control Channel Elements (CCEs) of the assigned control region within the subframe. 'TTI' is the sub-frame number and it's assigned to zero. 'PDCCHs' is the input port for placing the received PDCCHs train bits as whole on it .It can be variable for any number of bits. 'Res_uess_com' is the reset input port for assigning the initial assignments to each of ports. 'check6DCI1', 'check6DCI2' are output ports for indicating whether DCI control bits pass CRC check or not for the assumed DCI1 and DCI2 respectively, for each of $\mathrm{PDCCH}$ candidate location of PDCCH format 0 . 'check32DCI1' is an output port for indicating for each of the first $3 \mathrm{PDCCH}$ candidate locations of PDCCH format 1, determined from UESSS hashing function, whether the assumed DCI format 0/1A intended for UE or not. While 'check62DCI1' is an output port for indicating for each of the rest of $3 \mathrm{PDCCH}$ candidate locations of $\mathrm{PDCCH}$ format1.

Whether the assumed DCI format pass CRC check or not.

'Check32DCI2' is an output port for indicating for each of the first $3 \mathrm{PDCCH}$ candidate locations of PDCCH format 2, determined from UESSS hashing function, whether the assumed second DCI format, which depends on the configure transmission mode (RRC signaling), is intended for the UE or not. 'Check62DCI2' is an output port for CRC checking that is used for indication of the second DCI format for each of the rest of PDCCH candidate locations of PDCCH format 2. 'check4DCI1', 'check4DCI2' are output ports for defining whether DCI formats, that are transmitted using PDCCH format 2, are intended to the UE or not. 'Check8DCI1', 'Check8DCI2' are also output ports for indicating whether DCI formats, that are transmitted using PDCCH format 3, are intended to the UE or not.

Also, 'check4DCI1_common', 'check4DCI2_common' are output ports for CRC checking whether DCI formats transmitted using PDCCH format 2 in the first $2 \mathrm{PDCCH}$ candidate locations, defined from CSS hashing function, to the UE or not. 'check4DCI1_common3', 'check4DCI2_common3' are output ports for checking of the rest of PDCCH candidate locations of PDCCH format 2 in CSS.

Finally,'check8DCI1_common','check8DCI2_common' are the two last output ports for checking of any of DCI formats in the $2 \mathrm{PDCCH}$ candidate locations of $\mathrm{PDCCH}$ format 3 defined from CSS hashing function. The remaining variables are internal signals defined in the RTL. 'original6ctrl_bits22DCI1' is the signal port of the designed memory that stores the CRC checked control bits of DCI format intended to the UE. These DCI bits are obtained from the second half of PDCCH candidate locations of PDCCH format 1 . The remaining shown variables are internal signals of the designed memory to look in, if CRC check of any $\mathrm{PDCCH}$ candidate location indicates logic zero.

As shown from simulation, when 'check6dci1' indicates zero logic, it means successful decoding attempt and the DCI bits are stored in the internal signal 'original6ctrl_bitsDCI1'.As shown, it changed every change in the logic contained of 'check6dcil' that is depends on the result of decoding attempt.

\section{E. Timing Calculation of the Proposed Parallel Design}

As the maximum frequency resulted from the synthesis report for the proposed parallel design is $21.407 \mathrm{MHz}$ which is the inverse of the minimum clock period of $46.715 \mathrm{~ns}$. The time required for blindly decoding of one $\mathrm{PDCCH}$ candidate of PDCCH format 3 is $46.715 n s \times 51=2382.465$ $n s=2.3 \mu \mathrm{s}$. The proposed design blindly decodes each of PDCCH candidate locations for the two assumed DCI formats, simultaneously. Hence, one more PDCCH candidate needs to be checked for the two DCI formats. So, the total time required for blindly decoding of $\mathrm{PDCCH}$ candidates of PDCCH format 3 is only $2.3 \mu s \times 2=4.6 \mu \mathrm{s}$. This is the total required time to blindly decode $\mathrm{PDCCH}$ candidate locations of PDCCH format 3 defined from UESSS-CSS hashing functions. In similar manner, for $\mathrm{PDCCH}$ candidates of PDCCH format 2, defined from UESSS hashing function, the required time to accomplish the blind decoding process of 2 candidate locations of PDCCH format 2 is $4.6 \mu \mathrm{s}$. Similarly, for PDCCH candidate locations, defined from CSS hashing function, the required time is $4.6 \mu \mathrm{s}$ to accomplish the blind decoding process. This is resulted from dividing the monitoring process into two parts; each of it is responsible for decoding only two $\mathrm{PDCCH}$ candidate locations.

While, for PDCCH candidates which defined from UESSS of PDCCH format 1, there are 6 PDCCH candidate locations. In the proposed design, they are divided into two parts performing the blind decoding process concurrently. Each part blindly decodes only $3 \mathrm{PDCCH}$ candidate locations. With Viterbi decoder that is designed for PDCCH format 1 , the required number of clock cycles is 51 clock cycles. With minimum clock period of $46.715 \mathrm{~ns}$, so, for one blind decoding of each of PDCCH candidates of PDCCH format 1 , a time of $2.3 \mu \mathrm{s}$ is required. So, a total of $2.3 \mu \mathrm{s} \times$ $3=6.9 \mu \mathrm{s}$ is required for blind decoding of all of $3 \mathrm{PDCCH}$ candidate locations of PDCCH format 1 which is an aggregation of 2 CCEs.

For PDCCH candidates of PDCCH format 0 which is an aggregation of one CCE with payload size of 72 bits. So, the total number of clock cycles needed to accomplish Viterbi decoding process are 26 clock cycles, in addition to one more clock cycle for CRC check. Hence, the total time required for blindly decoding of one candidate location is $46.715 n s \times 27=1261.305 n s \approx 1.3 \mu \mathrm{s}$. With blind decoding of the assumed two DCI formats per PDCCH candidate at a time and, 6 PDCCH candidates locations, the total time for the complete blind decoding attempts is $1.3 \mu \mathrm{s} \times 6=7.8 \mu \mathrm{s}$.

As a result, depending on these calculation, a time of 7.8 $\mu \mathrm{s}$ which is equivalent to approximately of $11.7 \%$ of one LTE useful OFDM symbol time is required to accomplish the 44 blind decoding attempts and determines if there are 
DCI formats intended to the UE or it goes into micro sleep to save the limited battery power.

\section{F. Utilization Area and Power Consumption of the Proposed Parallel Design}

The resultant proposed parallel design is synthesized and implemented on virtex7 FPGA kit of part number XC7V2000T which is characterized by low power and large capacity. As shown from utilization area report in Fig. 12, the number of fully used LUT-FF pairs for the proposed parallel design is increased by only 1744 pairs compared to the proposed serial design.

\begin{tabular}{|l|r|l|r|r|}
\hline \multicolumn{5}{|c|}{ Device Utilization Summary (estimated values) } \\
\hline Logic Utilization & Used & Available & Utilization \\
\hline Number of Slice Registers & 63664 & 2443200 & $2 \%$ \\
\hline Number of Slice LUTs & 255450 & 1221600 & $20 \%$ \\
\hline Number of fully used LUTFF pairs & 9031 & 310083 & $2 \%$ \\
\hline Number of bonded IOBS & 634 & 850 & $74 \%$ \\
\hline Number of BUFG/BUFGCTRLs & 4 & 128 & $3 \%$ \\
\hline Number of DSP48EIs & 20 & 2160 & $0 \%$ \\
\hline
\end{tabular}

Fig. 12. Utilization area report of proposed parallel design.

The consumption power of the RTL is estimated using Xilinx Power Estimator (XPE) tool, as shown in Fig. 13.

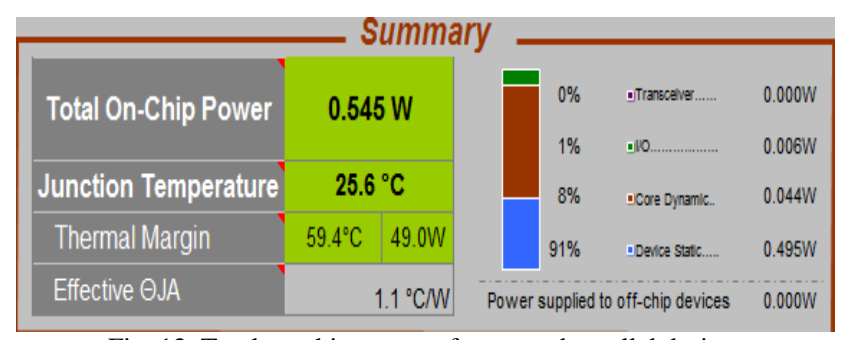

Fig. 13. Total on-chip power of proposed parallel design.

From Fig. 13, the leakage power is $495000 \mu \mathrm{w}$ of total on-chip power, while the dynamic power is $44000 \mu \mathrm{w}$ which is equivalent to only $8 \%$ of total on-chip power. The logic power is only $3 \%$ of total on-chip power which is equivalent to $17000 \mu \mathrm{w}$, while clock power is $26000 \mu \mathrm{w}$. The leakage, clock, logic and IO power consumption are shown in Fig. 14.

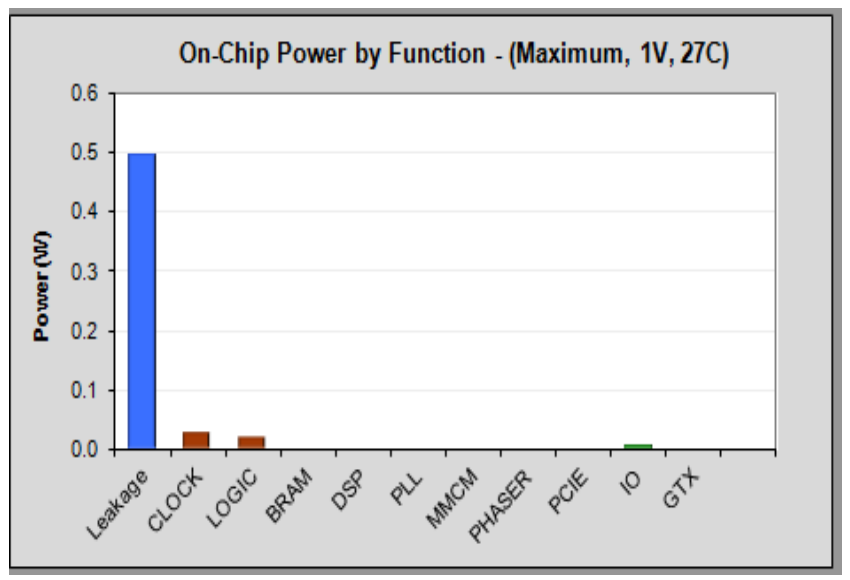

Fig. 14. Power consumption of clock, logic and IO.

\section{CONCLUSIONS}

Two designs of LTE DCI decoder are proposed in this paper. The proposed designs are simulated using Modelsim and implemented in Virtex7 FPGA kit. The proposed serial design is proved efficiency in decoding DCI associated with MBSFN subframes with extended cyclic prefixes, as a consequence of completing the blind decoding process within $87.3 \%$ of the whole OFDM symbol time. The proposed serial design is failed to meet the worst-case time of an LTE OFDM symbol with normal cyclic prefix. The proposed parallel design is presented and proved better performance in decoding time of DCI for all subframes. The proposed parallel design is proved efficiency of accomplishing 44 blind decoding attempts within a time of $7.8 \mu \mathrm{s}$, which is only $11.7 \%$ of an LTE useful OFDM symbol time, with a logic power consumption of $17000 \mu \mathrm{W}$ by increasing of only $5000 \mu \mathrm{w}$ compared to the proposed serial design. It is, also, proved efficiency in the occupied utilization area of Virtex7 FPGA kit.

\section{REFERENCES}

[1] L. Jialing, R. Love, K. Stewart, and M. E. Buckley, "Design and Analysis of LTE Physical Downlink Control Channel," in Proc. Vehicular Technology Conference 2009, VTC Spring 2009, IEEE 69th, 2009, pp. 1-5.

[2] J. Eriksson, R. Moosavi, and E. G. Larsson, "Complexity reduction of blind decoding schemes using CRC splitting," in Proc. IEEE Global Telecommunications Conference (GLOBECOM), 2012.

[3] F. Capozzi, D. Laselva, F. Frederiksen, J. Wigard, I. Z. Kovács, and P. E. Mogensen, "UTRAN LTE downlink system performance under realistic control channel constraints," in Proc. Vehicular Technology Conference Fall (VTC 2009-Fall), 2009 IEEE 70th, 2009, pp. 1-5.

[4] D. P. Malladi, J. Montojo, and S. Sarkar, "Methods and systems for PDCCH blind decoding in mobile communications," United States Patent Application Publication 2009/0168922, Jul. 2, 2009.

[5] R. T. Love, J. liu, V. Nangia, and R. Nory, "Control channel provisioning and signaling," Ed: WO Patent 2,010,129,400, 2010.

[6] M. Lauridsen, A. R. Jensen, and P. Mogensen, "Fast control channel decoding for LTE UE power saving," in Proc. Vehicular Technology Conference (VTC Spring), 2012 IEEE 75th, 2012, pp. 1-5.

[7] 3GPP TS 36.213, Evolved Universal Terrestrial Radio Access (EUTRA); Physical Layer Procedures (Release 8).

[8] E. Dahlman, S. Parkvall, J. Skold, and P. Beming, $3 G$ Evolution: HSPA and LTE for Mobile Broadband, Academic Press, 2010, ch. 16, pp. 352-362.

[9] S. J. Thiruvengadam and L. M. A. Jalloul, "Performance analyis of the 3GPP-LTE physical control channels," EURASIP Journal on Wireless Communications and Networking, vol. 2010, pp. 914-934, Nov. 2010.

[10] E. Dahlman, S. Parkvall, and J. Skold, 4G: LTE/LTE-Advanced for Mobile Broadband, Academic Press, 2011,ch. 10, pp. 195-200.

[11] Balamurali, "Optimal downlink control channel resource allocation for LTE systems," in Proc. 2010 International Conference on Signal Processing and Communications (SPCOM), 2010, pp. 1-5.

[12] F. Khan, LTE for 4G Mobile Broadband: Air Interface Technologies and Performance, Cambridge University Press, 2009, ch. 15 ,pp. 390396.

[13] 3GPP TS 36.212, Evolved Universal Terrestrial Radio Access (EUTRA); Multiplexing and Channel Coding (Release 8).

[14] M. Vestias, H. Neto, and H. Sarmento, "Sliding block viterbi decoders in FPGA," in Proc. 2012 22nd International Conference on Field Programmable Logic and Applications (FPL), 2012, pp. 595-598.

[15] M. Vestias and H. Sarmento, "Tradeoffs in the design of sliding block Viterbi decoders for MB-OFDM UWB systems," in Proc. 2012 IEEE International Conference on Consumer Electronics-Berlin (ICCEBerlin), 2012, pp. 173-177.

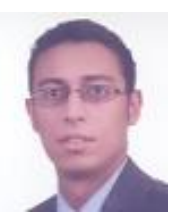

Mohamed S. Abo Zeid received his B.Sc. (honors) in Electrical Engineering from Tanta University in 2009.

$\mathrm{He}$ is currently a teaching assistant in Electronics and Electrical Communications department in Faculty of Engineering, Tanta University. 
Mohamed E. Nasr received his Ph.D. in Digital Communications Engineering from Delhi University in 1985 and his M.Sc. in Electrical Communications Engineering from Mansoura University in 1979 and B.Sc. (honors) in Computer Engineering from Menoufia University in 1975.

$\mathrm{He}$ is currently a professor of Wireless Communications in Tanta University. He was the head of Electronics and Electrical Communications department in Faculty of Engineering, Tanta University from 2004 to 2008.
Salah El Deen A. Khamis received his Ph.D. in Wireless Communications Engineering from Moscow Academy in 1992 and his M.Sc. in Communications Engineering from Menoufia University in 1985 and B.Sc. (honors) in Electrical Engineering from Cairo University in 1977.

He is currently an associate professor of Wireless Communications in Tanta University, the head of Electronics and Electrical Communications department in Faculty of Engineering, Tanta University. 